\title{
Research on Application of Overall Planning Graph in the Simulation of Equipment Maintenance
}

\author{
Ming Zhang ${ }^{1, a}$, Chunying $\mathrm{Qi}^{2, \mathrm{~b}}$, Jiang $\mathrm{Yu}^{2, \mathrm{c}}$ and Jiemin $\mathrm{Li}^{3, \mathrm{~d}}$ \\ ${ }^{1}$ Institute of Engineer Corps of PLA, Xuzhou 221004, China \\ ${ }^{2}$ Broadcasting and Television University of Xuzhou Jiangsu, Xuzhou 221001, China \\ ${ }^{3}$ Beijing Institute of Technology, Beijing 100081, China. \\ azhangming7929@sina.com
}

Keywords: Equipment maintenance; Assistant decision-making; Graph theory model.

\begin{abstract}
In this paper, overall planning graph, which is widely used in the process of equipment maintenance, is reinterpreted with the graph model. Then, this paper deeply discussed the various application of the graph theory model both in the equipment maintenance process and equipment purchase decision-making under complex conditions.
\end{abstract}

\section{Introduction}

Currently, in order to get an optimization scheme, overall planning graph is widely used to arrange maintenance resources in the process of equipment maintenance. But there are some disadvantages in most of the current overall planning graphs: graphs only consist of simple process diagrams; the constraint relations among processes are usually undefined; representation methods of graphs are not standardized; what is more, in numerical aspect, most overall planning graphs just simply described the orders and duration of every maintenance process without sufficient information, and thus that hinder the more in-depth study for maintenance process [1]. In this paper, the abovementioned imperfect overall planning graphs are called as the traditional graphs, and the overall planning graph described by graph theory model in this paper is called as the modern graphs. After the illustration of representation and calculation model of the modern overall planning graph, this paper also discusses how to use the modern overall planning graph to assist decision-making in equipment purchase.

\section{Model building}

According to the required equipments and the processes arrangement in the maintenance process, the premise constraint conditions in building the overall planning graphs are shown in Tab.1.

In graph theory ideas, modern overall planning graph should be shown as Fig.1, and it should be consisted in corresponding components [2]. Only in this way, we can sufficiently describe the relationship among the various maintenance processes and do more profound research.

Tab.1 Maintenance process and constraint conditions

\begin{tabular}{ccccc}
\hline Serial No. & Maintenance process & Frontal process & Equipment No. & Duration \\
\hline 1 & Disassemble the action parts & no & E1 & 4.0 \\
2 & Disassemble the transmission parts & no & E2 & 1.7 \\
3 & Test, maintain and replace the transmission parts & 2 & E3 & 2.0 \\
4 & Install the transmission parts & $1,2,3$ & E4 & 15.0 \\
5 & Debug the transmission parts & 4 & E5 & 4.8 \\
6 & Paint the vehicle body & 4 & E6 & 8.4 \\
7 & Trial run & 6 & E7 & 10 \\
\hline
\end{tabular}




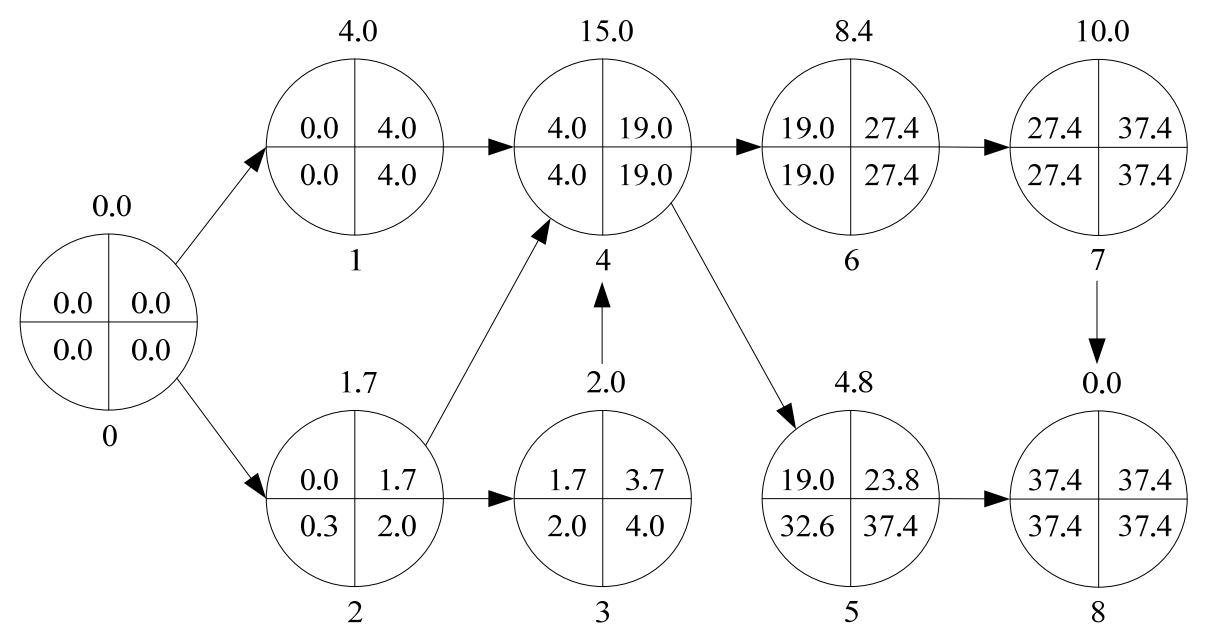

Fig.1 Overall planning g of the maintenance process

The meanings of four quadrants in Fig. 1 are as follows:

The number in the left upper quadrant is denoted by $e s_{i}$, represents the earliest starting time of No.i process.

The number in the right upper quadrant is denoted by $e f_{i}$, represents the earliest finishing time of No.i process.

The number in the left lower quadrant is denoted by $l s_{i}$, represents the at latest starting time of No.i process.

The number in the right lower quadrant is denoted by $l f_{i}$, represents the latest finishing time of No.i process.

The number above the node represents the average duration of every maintenance process, the number under the node represents the serial number of every maintenance process. No.0 and No. 8 separately represent the virtual starting node and the virtual finishing node [3].

$G_{i}$ is used as a set of all arcs which point to Node $i, Q_{j}$ is used as a set of all arcs which are pointed from Node $\mathrm{j}$, then, the numerical value relationship among the nodes are shown as model(1).

The value of every node in every quadrant could be calculated though repeated reiteration and searching calculation from positive order and reverse order as the model (1). As Fig.1, we know that: as for node 0 、 node 1 、 node 4 、node 6 、 node 7 , node $8, e s_{i}=l s_{i}, e f_{i}=l f_{i}$, that is to say, as for these processes, the earliest starting time equal to the latest starting time, the earliest finishing time equal to the latest finishing times. In order to ensure the shortest construction period of the whole project, these processes must begin and finish on schedule, they have not any flexible time. Therefore, the path connected with node 0 、 node 1 , node 4 , node 6 , node 7 , node 8 is called as critical path. The time needed for critical path is the shortest time to finish the whole equipment maintenance process [4].

$$
\left\{\begin{array}{l}
e s_{0}=e f_{0}=0 \\
e s_{i}=\max _{j \in G_{i}}\left\{e f_{j}\right\} \\
e f_{i}=e s_{i}+t_{i}(i=1,2, \cdots, 8) \\
l s_{i}=l f_{j}-t_{j} \\
\left.l f_{j}=\underset{i \in Q_{j}}{\min \left\{l s_{j}\right.}\right\} \quad(j=8,7,6, \cdots, 0) \\
l f_{8}=e s_{8}
\end{array}\right.
$$




\section{Multi-equipment model}

According to the calculation principle of critical path in graph theory, we know that, in modern overall planning graph, the time needed for critical path is the shortest needed time to finish maintenance for one equipment platform. But combined with model(1) and Fig.1, it only describes the static case of maintenance process for single equipment platform, the data and results reflected are still so limited. What the decision-makers more focused on is the maximum maintainability of the maintaining unit equipped with required equipments in a certain time under the case of task saturation, and how to improve their maintainability farthest at the least cost [5].

Under task saturation, there are always equipments required maintenance. For every node, especially for the start node, the next equipment immediately begin to be maintained following the last one's maintenance. At this time, according to the calculation principle of the graph theory, the starting time of next vehicle at the node is always affected by the anterior one, it means that, the $G_{n i}$ includes not only the anterior nodes related to the equipment, but also the anterior equipment's maintenance nodes. In the same way, ${ }^{n j}$ could increase the influence on the anterior equipment.

At this time, the virtual start Node 0 and the virtual finish Node 8 are apparently not affected, the relationship between the starting time and the finishing time of one node is not affected too, however, the numerical relationship between different nodes would change. While repairing No.n equipment, the calculation model of every node is shown as model (2):

$$
\left\{\begin{array}{l}
e s_{0}=e f_{0}=0 \\
e s_{n i}=\max _{j \in G_{n i}} \quad\left\{e f_{j}, e f_{(n-1) j}\right\} \quad(i=1,2, \cdots, 8) \\
e f_{n i}=e s_{n i}+t_{i} \\
l s_{n j}=l f_{n j}-t_{j} \\
l f_{n j}=\min _{i \in Q_{n j}} \quad\left\{l s_{n i}, l s_{(n-1) i}\right\} \quad(j=8,7,6, \cdots, 0) \\
l f_{n 8}=e s_{n 8}
\end{array}\right.
$$

When the batch of equipments begin to be repaired, decision-makers would not focus on the critical paths of single equipment or multi-equipments, what they concern is when these equipments be finished maintaining and could be used to fight again. Some conceptions about time, such as $l s_{n i}$ and $f_{n i}$, become no longer important. For this reason, both $l s_{n i}$ and $f_{n i}$ would be omitted in this paper. From the model (2), we know that the finishing time of No.n equipment is $e f_{n 8}$. In fact, for a maintenance unit, when $e f_{n 8}=f_{n 8} \leq 60$, max. $n$ is the maximum sum of equipments permitted to be maintained in a limited time [6].

\section{Equipment purchase decision-making model}

In the case of given funds, how to add new equipments to ensure short maintenance time at the largest degree and to improve the units' maintainability? Then, we can introduce the expenses of every equipment and find out various combinations, then, we use the model (2) to verify the ultimate result of the combinations. The combination with the maximum sum of equipments permitted to be maintained in the limited time is the best purchase decision of equipments [7].

First of all, we should build the integer programming model according to the integer programming theory, and then, based on the constraints mentioned above, we can obtain the corresponding solution set.

Suppose that there are $t$ kinds of equipments in the whole maintenance process, the price of No. 1 equipment is $a_{l}$, the fund given for purchase is $\mathrm{M}$, if we purchase $Z_{l}$ sets of No. 1 equipment. The purchase plan is shown as an integer programming model which is built by the first two formulas in the model(3) with constraint conditions [8]. 


$$
\left\{\begin{array}{l}
\sum z_{l} a_{l} \leq M \\
z_{l}>0(l=1,2, \cdots, t) \\
e s_{n i}=\max _{j \in G_{n j}}\left\{e f n_{j}\right\}(n \leq m) \\
e s_{n i}=\max \left\{e f_{n j}, \min \left(e f_{n-k}\right)\right\}(n \geq m) \\
(k=1,2, \cdots, m)
\end{array}\right.
$$

Combination of sets of equipments required purchase could be obtained though using the integer programming model, next, substitute $Z_{l}$ into model(3) to calculate, when the $e f_{n i}$ is close to the given time, the scheme with the maximum $\mathrm{n}$ (sum of sets of equipments permitted maintained) is the solution.

\section{Summary}

Currently, there are many models adopted to study equipment maintenance support. [4-9]In these theories, maintenance unit are regarded as a whole object, however, from the current situation, it is unpractical to add several maintenance units to the whole object. If we research the maintenance process from the relations among the processes in maintenance units, and improve the maintenance units within, we could short the maintenance time and enhance the equipment support levels, that not only meet the efficiency requirement of maintenance units, but also improve the support ability of maintenance units to satisfy the demand of higher authorities.

Besides for the equipment purchase decision-making, this model could be used to arrange all maintenance support resources, such as the number collocation among different types of workers, the proportion of different grades of maintenance personnel, and so on.

\section{References}

[1] Zhang M, Su H J, Yuan L, et al. Research on Problems and Basal Theory of Engineering Equipment's Maintainability Test[C]//Advanced Materials Research. 2011, 328: 2446-2449.

[2] Wang Zilan, Wang Ciguan, Research on the management scheme of the locomotive based on statistics and design method[J]. Railway transportation and economy, 2012,34(1):82

[3] Zhang M, Yu J, Qi C Y, et al. Research on Evaluation Model of Engineering Equipment Supportability in Operating Phase[C]//Advanced Materials Research. 2012, 403: 3652-3655.

[4] Chen Qinghua. Operational Research of The Equipment[M].Beijing: National Defence Industry Press, 2005

[5] Zhang M, Qi C Y, Yuan L, et al. Research on Assessment Method of Engineering Series Equipment Assignment Plan[C]//Advanced Materials Research. 2012, 403: 3334-3338.

[6] Yang Weimin. Pandect of Dependability、 repairable、 indemnificatory[M].Beijing: National Defence Industry Press, 1995

[7] Zhang M, Shi Z, Dou C, et al. Research on Lash-up Maintenance Technology of Engineering Equipment[C]//2012 National Conference on Information Technology and Computer Science. Atlantis Press, 2012.

[8] Zhang M, Yu J, Wang M H, et al. Study on Design of Intelligent Decision Support System of Engineering Equipment Repair[C]//Applied Mechanics and Materials. 2012, 127: 36-41. 